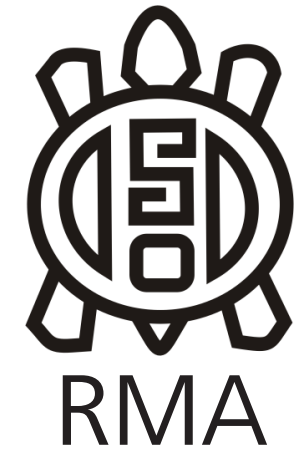

Dossier

\title{
Circulación humana durante el Holoceno tardío en el bosque y ecotono bosque-estepa: el curso inferior del río Penitente (suroeste de Santa Cruz)
}

Human circulation during the Late Holocene in the forest and the forest-steppe ecotone: the lower Penitente River

(Southwest Santa Cruz)

Juan Bautista Belardi*, Flavia Carballo Marina** y Luis Alberto Borrero***

* Universidad Nacional de la Patagonia Austral. Unidad Académica Río Gallegos. ICASUR - CONICET. Piloto Leo Rivera s/n Campus Universitario. (9400) Río Gallegos, Santa Cruz. E-mail: juanbautistabelardi@gmail.com

** Universidad Nacional de la Patagonia Austral. Unidad Académica Río Gallegos. ICASUR. Piloto Leo Rivera s/n Campus Universitario. (9400) Río Gallegos, Santa Cruz. E-mail: flaviacarballomarina@gmail.com

*** IMHICIHU-CONICET. Saavedra 15. (C1083ACA) y Universidad de Buenos Aires, Ciudad Autónoma de Buenos Aires. E-mail: laborrero2014@gmail.com

\begin{abstract}
Resumen
El curso inferior del río Penitente se ubica en el sur de la zona de los morros -nacientes del río Gallegos- y es muy poco conocido desde la arqueología. El registro arqueológico de la terraza de la margen este del río muestra tipos artefactuales y materias primas líticas que señalan la interacción con núcleos poblacionales ubicados al noroeste y al este del área de estudio, y con el mar de Otway al sur, durante el Holoceno tardío. La distribución del registro arqueológico es relativamente continua a lo largo del río y da cuenta de una diversidad de contextos que difieren en intensidad y funcionalidad. Así, el conocimiento de esta cuenca añade variabilidad al registro arqueológico del sector de los morros y se brindan nuevas evidencias sobre el uso humano del bosque, relacionado con el reparo que ofrece tanto bajo condiciones de circulación como de mayor permanencia.
\end{abstract}

Palabras clave: Holoceno tardío; Bosque; Circulación; Interacción; Tecnología lítica.

\begin{abstract}
The lower segment of the Penitente River is located south of the area of the morros -upper Rio Gallegos basin- and it is not very well known from an archaeological perspective. The archaeological record from the eastern bank of the river presents tools and lithic raw materials indicating Late Holocene interaction with populational nodes located northwest and east, and also with the Otway Sound. The distribution of the artifacts is relatively continuous along the river and informs about a diversity of archaeological contexts that differ in intensity of occupation and function. Then, the perspective provided by the Penitente basin, beyond the addition of new evidences about the human use of the forest-mainly its offer of sheltered conditions for both circulation and permanence-increases the recorded archaeological variability of the area of the morros.
\end{abstract}

Keywords: Late Holocene; Forest; Circulation; Interaction; Lithic technology.

\section{Introducción}

El curso inferior del río Penitente (148 a 119 msnm en el área de estudio) se localiza al suroeste de la provincia de Santa Cruz y es muy poco conocido desde la arqueología (Molinari 2000). El río nace en la cordillera Vidal (República de Chile), desconectado del Campo de Hielo Patagónico Sur (CHPS), lo que resulta en una estacionalidad poco significativa. Lo señalado habría permitido que las poblaciones cazadoras tuvieran un acceso al bosque de ñire (Nothofagus antarctica), más extenso hasta tiempos recientes, sumamente sencillo de circular por la topografía suave y utilizable durante lapsos amplios.

El río recorre -en sentido sud-nornoroeste- $18 \mathrm{~km}$ por territorio argentino atravesando el ecotono bosqueestepa graminosa, que posee una capacidad de carga de herbívoros relativamente alta (Mazzoni y Vazquez 2001). En el paraje Puente Blanco se une con el río Rubens para dar nacimiento al río Gallegos (Figura 1). El río atraviesa 
depósitos sedimentarios de origen glacial y glacifluvial de bajo relieve relativo (Díaz et al. 2015). Su planicie aluvial muestra un gradiente extremadamente bajo, con meandros abandonados ocupados por lagunas en medialuna (Mazzoni 2017). Sobre su margen este, ha disectado una morena de fondo sobre la que se labró una terraza (120 a $132 \mathrm{msnm}$ ) que ha expuesto rodados de variada litología y tamaños, aptos para la talla. Tiene un régimen pluvio-nival. El invierno y la primavera son las estaciones con caudales medios más elevados; tiene una variabilidad interanual muy significativa (Díaz et al. 2015). En la actualidad, por su bajo caudal puede cruzarse a pie o vadearse sin dificultad (Borrero y Borrazzo 2011).

Las nacientes de la cuenca del río Gallegos poseen un rasgo distintivo del paisaje dado por cuatro cuellos volcánicos (400 msnm), últimas manifestaciones del Campo Volcánico de Pali Aike (CVPA). Son los morros Chico (Chile), Philippi, Domeyko y Gay (Argentina) (Figura 1). Este sector es el más obstrusivo de la cuenca (Borrero 1994-1995), por lo que el paisaje es muy legible (Golledge 2003). Esto sustenta la propuesta de que los morros habrían actuado como hitos que orientaran la circulación de las poblaciones humanas (Borrero et al. 2011). Para momentos históricos el área ha sido propuesta como ruta natural de penetración desde el sur y desde el oeste hacia el interior de Patagonia (Borrero y Borrazzo 2011; Charlin et al. 2011).

Hacia el este y el oeste de los morros se han reconocido dos zonas cuyos paisajes arqueológicos reflejarían la existencia de dos núcleos poblacionales con rangos de acción no superpuestos y bien definidos, que difieren notablemente en las frecuencias artefactuales y en sus características tecnológicas líticas. El primer núcleo, situado al noroeste del río Penitente, abarca la costa sur del lago Argentino, la cordillera Baguales, Fuentes del Coyle, los ríos Guillermo y Primavera, la estribación oeste de la Cordillera Chica y Puesto Aserradero (Argentina) y la región de Última Esperanza (Chile). El segundo, hacia el este, comprende el interior de CVPA -cursos medios de los ríos Gallegos y Chico- y tiene conexiones con la costa atlántica y el estrecho de Magallanes (Borrero 2015, Figura 1; Charlin et al. 2011; Pallo et al. 2020). A diferencia de ambas zonas, en los morros las frecuencias artefactuales descienden notablemente, lo que llevó a que fuera considerada como una zona vacía (Gómez Otero 1991). Hoy, a 30 años de esa propuesta, se sabe que no lo ha sido. Pero que aun así, su impronta arqueológica dista de ser fuerte, ya que la señal en cercanía de los morros es más leve, tardía y vinculada a un uso logístico del espacio (Charlin 2012; Charlin et al. 2011; Borrero 2015; Borrero y Borrazzo 2011; Borrero et al. 2011; L'Heureux y Borrero 2016; Pallo et al. 2020). El curso inferior del río Penitente forma parte del sector sur de los morros y prácticamente carecía de antecedentes (Molinari 2000). Así, conocer su paisaje arqueológico complementa y amplía la discusión del uso local del espacio por parte de las poblaciones humanas y de los niveles de interacción con ambos núcleos poblacionales.

Con el fin de poner en perspectiva los trabajos realizados, se presenta el marco ambiental y arqueológico regional del sector de los morros. Luego, se describe la información según los sectores de relevamiento: Puente Blanco, Ea. Rincón de Los Morros, Ea. Rincón de Los Morros-Lagunas y Bosque de Las Bandurrias. El registro arqueológico ubicado en la terraza de la margen este del río exhibe

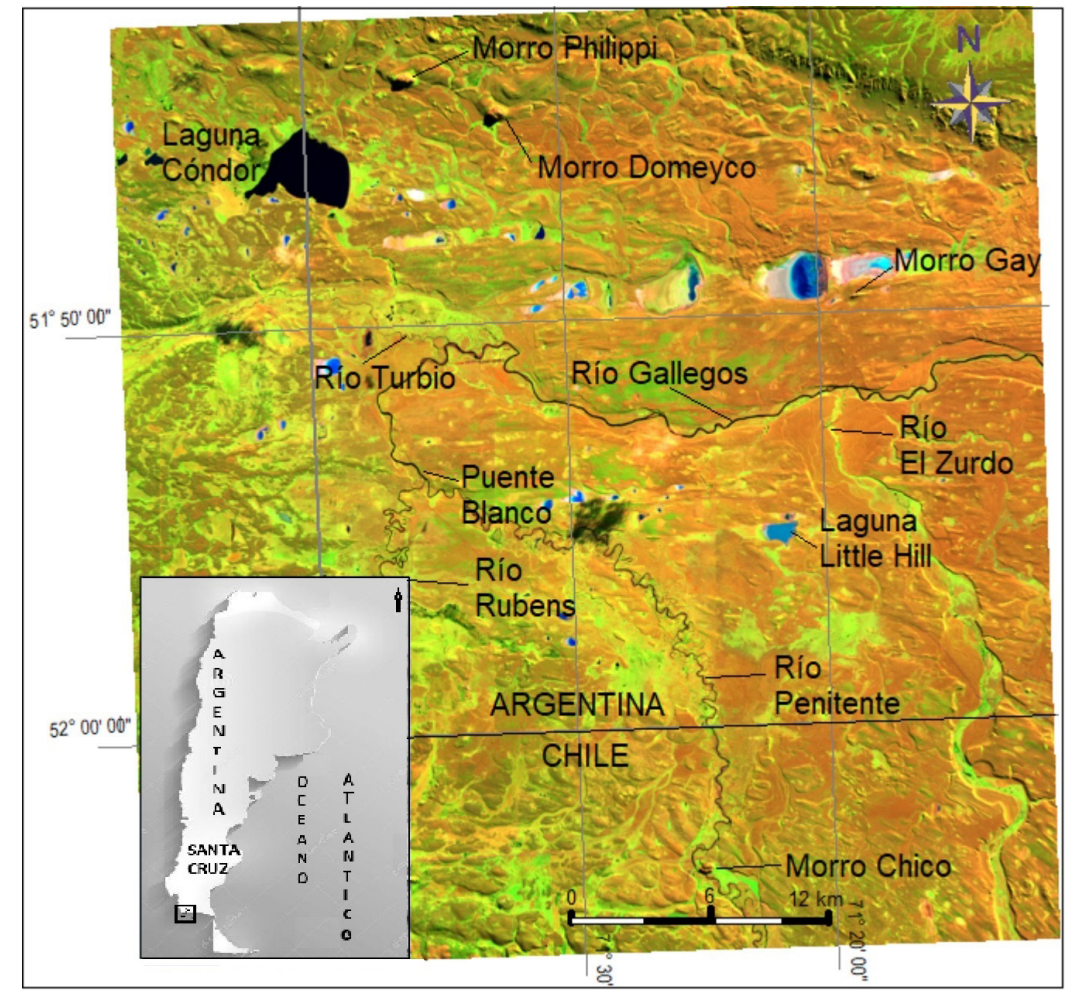

Figura 1. Mapa de la región de estudio.

Figure 1. Map showing the study area. 
elementos que revelan interacción con aquellos espacios articulados por los dos rangos de acción mencionados y con el mar de Otway, a unos 100 km lineales hacia el sur. Hay una relativa continuidad en la distribución espacial de artefactos y arqueofaunas a lo largo de dicha terraza y una mayor diversidad de contextos. Además de hallazgos aislados se infieren espacios de actividades limitadas y otros con un mayor componente residencial. Estos resultados añaden variabilidad al sector de los morros, brindan nuevas evidencias sobre el uso humano del bosque en el extremo sur de Santa Cruz durante el Holoceno tardío (Borrero y Borrazzo 2011; Carballo Marina et al. 2016; Charlin et al. 2011; Pallo y Borrero 2015) y sitúan al curso inferior del río Penitente en el marco mayor de la arqueología del sur de Patagonia continental.

\section{Antecedentes ambientales y arqueológicos regionales}

Los datos polínicos indican que el bosque está presente en la región donde se encuentra el río Penitente desde ca. 3500 años AP (Schäbitz 1991), alternándose entre bosque abierto y cerrado (Huber y Markgraf 2003; Huber et al. 2004). Las temperaturas entre 3000 años y 1000 años AP fueron semejantes o aún mayores a las actuales (Schäbitz 1991). Para el río Rubens se ha documentado un bosque pleno entre ca. 5100-2400 años AP, lo que también ocurre en Vega Ñandú y otros sitios, en Torres del Paine (Chile) (Huber et al. 2004; Moreno et al. 2009). Columnas polínicas sostienen un cuadro de variaciones en la intensidad y/o posición latitudinal del borde sur de los westerlies (vientos del oeste) que reflejan incrementos en las precipitaciones durante la Pequeña Edad del Hielo (PEH). En los Andes patagónicos los máximos avances glaciarios durante este periodo fueron datados dentro de los siglos XVII y XIX (Glasser et al. 2004; Garibotti y Villalba 2009) y produjeron un neto desplazamiento del ecotono bosque-estepa hacia el este (Moreno et al. 2009: 37). A pesar de la tendencia hacia un paulatino incremento de la temperatura posterior a los avances neoglaciales que resultaron en una reducción del bosque (Mancini 2002), una cobertura arbórea más extendida caracterizaría el corto lapso de las ocupaciones aquí discutidas, precisamente por su superposición al menos parcial con la PEH. Es esperable que estos límites orientales del bosque no tuvieran características que los pudieran erigir en barreras; por el contrario, harían más habitables ciertos sectores del espacio aún durante las estaciones más frías.

El ñire, que es la especie predominante en el río Penitente, se adapta a gran variedad de condiciones ambientales (precipitaciones anuales entre 300 y $800 \mathrm{~mm}$ y resiste heladas de hasta $-22^{\circ} \mathrm{C}$ ), lo que determina su amplia distribución espacial (Peri y Ormaechea 2013). El bosque actual en el curso medio del río es de ñires bajos, se encuentra fragmentado y se desarrolla sobre la terraza.
La información proveniente de la zona de los morros se relaciona en primer lugar con contextos funerarios. Sobre el morro Chico (Figura 1) se recuperó un esqueleto femenino con deformación tabular erecta y evidencias de consumo de dieta mixta fechado en $480 \pm 30$ años AP (Alfonso-Durruty et al. 2015). Estaba asociado a un colgante confeccionado en lignito con motivos de trazos paralelos incisos atribuibles al "Estilo Río Chico", ocre rojo y 12 lascas de obsidiana verde provenientes del mar de Otway. El diseño del colgante es semejante a la decoración de piezas óseas recuperadas en los sitios de Englefield y Bahía Buena, en el mar de Otway y sobre el estrecho de Magallanes, respectivamente (Prieto 1984). Por su parte, en la cima del morro Philippi se encontró un enterratorio tipo chenque que contenía los esqueletos de tres niños pequeños, grandes bifaces de sílex y boleadoras pintadas con ocre rojo y restos de un molusco (Ortiz Troncoso 1973).

Al suroeste del morro Philippi, en el ecotono bosqueestepa, se encuentra la laguna Cóndor (Figura 1). Sobre su margen noreste se registró una amplia distribución de restos faunísticos y artefactos líticos. Se constató la explotación principal del guanaco (Lama guanicoe) y en forma ocasional la del huemul (Hippocamelus bisulcus) y el caballo (Equus caballus) (L'Heureux y Borrero 2016). Dentro de las rocas empleadas se destacan por su abundancia las Rocas de Grano Fino Oscuras (RGFO) (Charlin 2005) tipo Potrok Aike (RGFO PKA). La fuente más cercana de esta roca se localiza en la laguna Potrok Aike (centro del CVPA) a unos $88 \mathrm{~km}$ hacia el este del río Penitente. También se relevaron 10 artefactos de obsidiana gris verdosa veteada que vincularía a la laguna con espacios ubicados hacia el noroeste (cordón Baguales) y con el CVPA, por ser la obsidiana más representada en esa zona. Además, se registraron dos lascas de obsidiana verde (Charlin 2012, Tabla 1) y un lito discoidal que podría tratarse de una preforma de piedra horadada tal como la registrada en Ea. Morro Chico (Borrero y Borrazzo 2011, Figura 1). Se han inferido tareas de reactivación de instrumentos que habrían ingresado manufacturados al sitio y descartados en otros espacios $y$, en menor proporción, la manufactura y el mantenimiento de artefactos también transportados (Charlin 2012). Las dataciones obtenidas $-220 \pm 41$ años AP y $187 \pm 41$ años AP (Charlin et al. 2011) y $213 \pm 43$ años AP y 232 \pm 43 años AP (L'Heureux y Borrero 2016)- muestran ocupaciones durante momentos tardíos; a las que se suma la presencia de materiales históricos. La laguna se habría usado en forma estacional y logística -articulada desde el este-, de manera recurrente pero por períodos cortos.

Pallo et al. (2020) presentaron un análisis sobre la distribución de grandes láminas de lutita que han sido empleadas como formas base para la confección de raederas y cuchillos, ya sean de un filo o doble convergentes y que pueden incluir filos cortos en sus extremos. La mayor frecuencia de estas piezas junto 
con la existencia de variadas fuentes potenciales de lutita de tamaños grava y bloque entre Ea. La Verdadera Argentina y laguna Larga, permitieron reconocer a estos artefactos como indicadores del rango de acción del núcleo poblacional reconocido al noroeste de la zona de los morros, por lo que las autoras proponen que pueden ser empleados para discutir conexiones espaciales y mecanismos de interacción entre poblaciones. Los conjuntos de raederas sobre hojas de lutita y la fuente potencial de esta roca más cercana al río Penitente fueron identificados en Puesto Aserradero (Charlin et al. 2011) y en laguna Larga (Pallo et al. 2020, Figura 3), a unos 30 y $20 \mathrm{~km}$ hacia el noroeste del paraje Puente Blanco.

La información arqueológica del sector argentino del río Penitente (Figura 1) también era muy acotada. Sobre la terraza de la margen este, inmediatamente al este del casco de la Ea. Rincón de los Morros, Molinari (2000) realizó un relevamiento sumario de materiales arqueológicos en superficie y en estratigrafía en cinco hoyadas de deflación contiguas que denominó Rincón de Los Morros 1. El registro se focalizó sobre los artefactos formatizados, entre los que predominan las raederas. En el interfluvio Penitente-El Zurdo se ubica la laguna Little Hill (Figura 1); en su margen norte se recuperaron artefactos con filos largos en dacita y RGFO (Charlin 2005). A pesar de ser una muestra pequeña, existe gran variabilidad artefactual. Asimismo, se registró un asta de huemul -un animal de bosque- que no presentó modificaciones antrópicas (Carballo Marina et al. 2008). El registro más reciente de ocupaciones corresponde al campamento histórico del cacique Mulato (Childs 1936, Martinic et al. 1995), en el sector chileno del río El Zurdo (Figura 1). También los relatos de viajeros refieren al cruce de este río (Dixie 1996) y a la circulación por las cabeceras del río Gallegos (Greenwood 2015; Rogers 2002 -1877; ver un desarrollo detallado en Charlin et al. 2011).

La información sobre la zona de los morros está vinculada con contextos mortuorios y otros, que, como la laguna Cóndor, habrían sido articulados de manera logística. Por estos motivos, los morros son vistos como alejados de áreas centrales y/o de uso más intensivo como la correspondiente a los núcleos poblacionales propuestos hacia el noroeste y Pali Aike (Borrero 2015).

\section{Metodología}

A partir de estudios distribucionales (Foley 1981; Dunnell y Dancey 1983, entre otros) y dentro de un esquema exploratorio se buscó caracterizar el paisaje arqueológico del curso inferior del río Penitente. Se muestrearon las ventanas de visibilidad que ofrece el bosque: hoyadas de deflación, cicatrices de erosión y, hacia la estepa, los márgenes de lagunas y sectores antropizados como corrales y caminos. En estas superficies se implementaron transectas dirigidas con unidades de muestreo de dimensiones variables que fueron registradas mediante coordenadas geográficas. Se recolectaron todos los materiales observados.

Los relevamientos se focalizaron sobre las hoyadas de deflación y cicatrices de erosión formadas en la superficie de la terraza de la margen este del río. La misma fue recorrida desde el norte del paraje Puente Blanco (120 msnm) hasta el sitio Rincón de Los Morros 1 (RM1) (132 msnm) -que es el mismo que fue identificado por Molinari (2000) (Figura 2)-, donde finaliza la distribución actual del bosque sobre la terraza. Desde el sitio se realizó una transecta de 1,5 km, hacia el este bordeando la terraza sin que se produjeran hallazgos. Luego se tornó al norte, ya en la estepa graminosa, hacia el extremo este de una serie de siete lagunas (Figura 2) y desde allí se llevó a cabo una transecta lineal continua de siete $\mathrm{km}$ hacia el oeste que enhebra a las lagunas. Sólo sus márgenes tienen buena visibilidad arqueológica y se registró una muy baja frecuencia de hallazgos. Hacia el sur, se llegó al límite internacional. Este sector, que se encuentra por debajo de la mencionada terraza, también corresponde al ecotono bosque-estepa aunque los parches de bosque se encuentran más raleados que en el espacio comprendido entre Puente Blanco y Rincón de Los Morros 1. Las observaciones fueron sumarias y se realizaron en cicatrices de erosión vinculadas a meandros abandonados y al borde de la terraza del rio.

Los sectores de relevamiento, basados sobre la buena visibilidad arqueológica, fueron denominados de acuerdo con su proximidad espacial con parajes (Puente Blanco), estancias (Rincón de los Morros) o con aquellos nombrados por sus dueños (Bosque de las Bandurrias). Los sitios y espacios de recolección de materiales se determinaron sobre la base de la alta densidad (orden de magnitud $10^{-1} \cdot \mathrm{m}^{2}$ ) de materiales líticos y restos óseos mientras que los hallazgos aislados son referidos a la concentración artefactual más cercana, distancia que varía entre 20 y 100 m respecto de la misma. Se realizaron recolecciones de materiales líticos y arqueofauna y, en el caso de RM1, se hizo un sondeo sobre un perfil expuesto con artefactos líticos en su base y se tomaron muestras de restos de guanaco de otro perfil expuesto para su datación.

El estudio de los artefactos líticos se llevó a cabo siguiendo los lineamientos propuestos por C. Aschero $(1975,1983)$ y considerando las siguientes variables: tipo artefactual, materia prima empleada y calidad para la talla (sensu Aragón y Franco 1997), estado, dimensiones, presencia de talón y, para el caso de los artefactos formatizados, su tipo, forma base y características generales. Se contabilizaron sólo los desechos de talla con talón. La determinación de las rocas se realizó en forma macroscópica.

Dado que la mayor parte de los artefactos recuperados son líticos, y por razones de espacio, se privilegió su análisis por sobre el de los restos arquefaunísticos. Así, 
se realizó una caracterización general del conjunto de restos de guanaco: determinación del NISP, perfil de meteorización y relevamiento de huellas y marcas (Mengoni Goñalons 1999).

En la Tabla 1 se enumeran los lugares trabajados -señalados en la Figura 2-, las superficies relevadas, las frecuencias de desechos de talla sin talón y de tipos de artefactos por materia prima lítica. En la Tabla 2 se comparan los conjuntos de $\geq 10$ artefactos, sobre la base de la densidad artefactual por $\mathrm{m}^{2}$, riqueza, relación entre núcleos/ desechos de talla, desechos de talla externos/ desechos de talla internos y artefactos formatizados/ desechos de talla. Si bien en estas relaciones no se discrimina por materia prima lítica, esto permite inferir tendencias generales y establecer diferencias en las actividades desarrolladas. Además, la tabla muestra los porcentajes de las tres rocas más utilizadas.

\section{Resultados}

La presentación de la información se orienta de norte a sur (Figura 2). La visibilidad del material arqueológico es resultado de un patrón sesgado por procesos morfodinámicos, ya que fue localizado, mayoritariamente, en la superficie de la terraza. Esta es afectada por erosión eólica e hídrica, generado hoyadas de deflación y cicatrices de erosión que dejan expuestos artefactos líticos y restos óseos (Figura 3 A-D). A ello se suma el pisoteo del ganado de las estancias, actividad aún vigente en esta región. El talud de la terraza tiene una pendiente de $\geq 60^{\circ}$ y carece de vegetación, lo que favorece la reptación de los materiales arqueológicos. A la vez, expone rodados de variada litología y de buena y muy buena calidad para la talla; lo mismo se ha verificado en los bordes de las lagunas, aunque en menor frecuencia. Se identificaron rodados de dacita y RGFO de distintos tamaños que condicen con los módulos de los artefactos registrados. Las observaciones replican resultados alcanzados por Charlin (2009), quien realizó muestreos de materias primas líticas en un depósito de till en la margen derecha del río Gallegos, en el paraje Puente Blanco. Estos indicaron la disponibilidad mayoritaria de dacita, seguida por las rocas sedimentarias, algunas de ellas lutitas, aunque en nódulos pequeños (Charlin 2009). En el río Zurdo también predominan las dacitas seguidas por los basaltos y las andesitas. Además, se han recuperado RGFO volcánicas y sedimentarias. Se realizó un corte delgado a este último tipo y fue determinada como lutita (Charlin 2009). Aunque en baja frecuencia y calidades inferiores se hallan nódulos chicos de RGFO PKA, en la confluencia de los ríos Zurdo y Gallegos (Charlin 2009; Cirigliano et al. 2020). Estos resultados muestran la alta disponibilidad de rocas aptas para la talla en escala regional y, en especial, a lo largo de los ríos.

\section{Paraje Puente Blanco}

En la superficie de la terraza de la margen este de la desembocadura del río, en hoyadas de deflación formadas entre manchones de ñires, se reconocieron artefactos tanto aislados como concentrados al aire libre y en superficie. Los sitios fueron denominados Puente Blanco 1 a 6 y Perfil caído (Tabla 1, Figura 2, Figura 3 A y B). A los procesos naturales que afectan a la terraza del río, se suma una circulación de personas por actividades recreativas que es mayor aquí que en otros sectores de la cuenca.

El conjunto recolectado en el sitio Puente Blanco 6 (PB6) (Tablas 1 y 2) se compone de lascas internas en RGFO PKA, con talones lisos. Dos de ellas de reducción bifacial. Uno de los hallazgos aislados corresponde a una raedera simple de lutita negra sobre hoja (103 x $41 \times 10 \mathrm{~mm})$ (Figura 4 B 5).

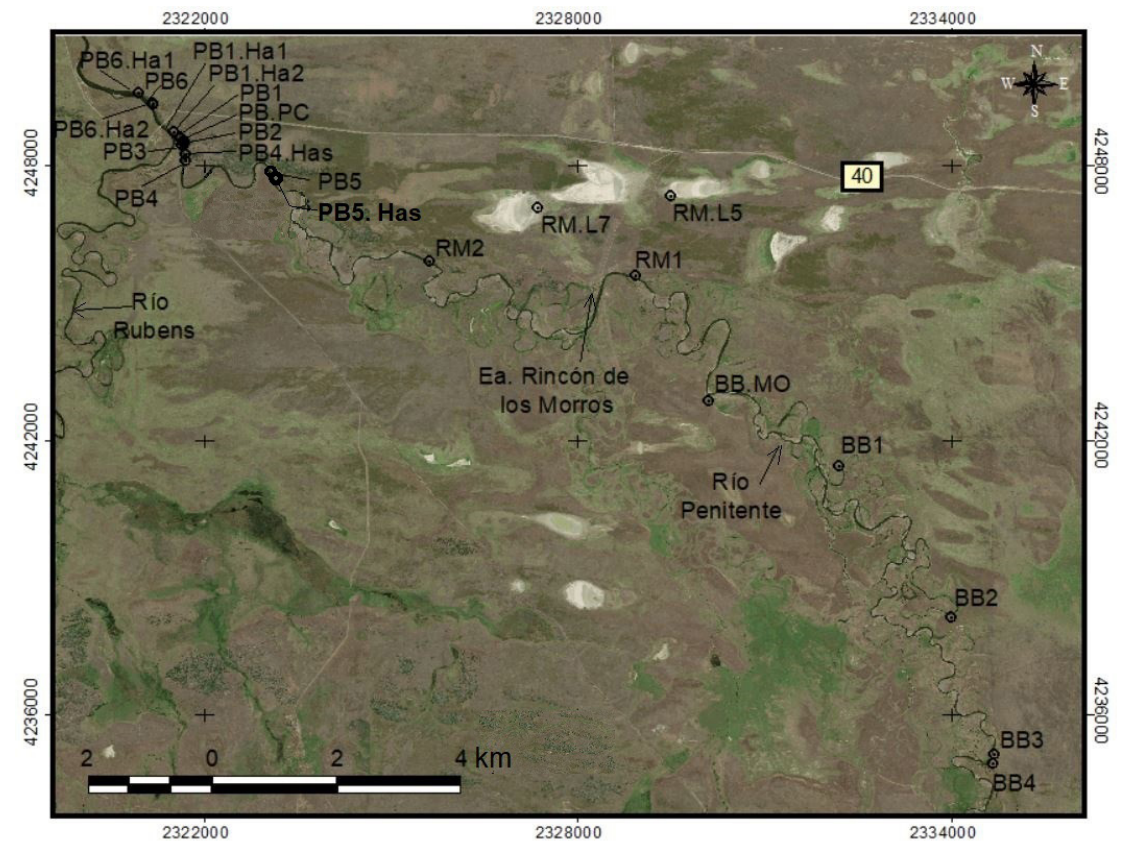

Figura 2. Lugares trabajados. Referencias: PB: Puente Blanco, PB PC: Puente Blanco Perfil caído, Ha.: hallazgo aislado, RM: Rincón de Los Morros, RML: Rincón de Los Morros-Lagunas, MO: margen oeste, BB: Bosque de Las Bandurrias.

Figure 2. Worked places. References: $P B$ : Puente Blanco, PB PC: Puente Blanco Perfil caído, Ha.: isolated find, RM: Rincón de Los Morros, RML: Rincón de Los MorrosLagunas, MO: West margin, BB: Bosque de Las Bandurrias. 


\begin{tabular}{|c|c|c|c|c|c|c|c|c|}
\hline \multicolumn{9}{|c|}{ Puente Blanco 6. Superficie relevada: $2 \mathrm{~m}^{2}$ (desechos de talla sin talón, $n=2$ ) } \\
\hline \multirow[t]{2}{*}{ Tipo artefactual } & \multicolumn{8}{|c|}{ Materias primas líticas } \\
\hline & \multicolumn{8}{|c|}{ RGFO } \\
\hline L. externa & \multicolumn{8}{|c|}{2} \\
\hline L. interna & \multicolumn{8}{|c|}{8} \\
\hline \multicolumn{9}{|c|}{ Puente Blanco 6. Hallazgos aislados. Superficie relevada: $500 \mathrm{~m}^{2}$} \\
\hline \multirow[t]{2}{*}{ Tipo artefactual } & \multicolumn{8}{|c|}{ Materias primas líticas } \\
\hline & \multicolumn{3}{|c|}{ RGFO (¿lutita negra?) } & \multicolumn{5}{|c|}{ Dacita } \\
\hline Raedera & & 1 & & & & 1 & & \\
\hline & Puente & anco 1. & ficie relevada: & $4 \mathrm{~m}^{2}$ (des & de talla & talón, $n=$ & & \\
\hline Tipo artefactual & & & Materi & primas lit & & & & 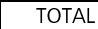 \\
\hline & Dacita & RGFO & Calcedonia & Granito & Sílice & Lutita & Indet. & \\
\hline L. externa & 8 & 3 & 1 & 3 & --- & --- & 2 & 17 \\
\hline L. interna & 10 & 8 & 1 & 1 & 1 & --- & 5 & 26 \\
\hline Núcleo & 4 & 1 & --- & --- & --- & --- & --- & 5 \\
\hline Raspador & --- & 1 & 2 & --- & --- & --- & --- & 3 \\
\hline Cuchillo & 1 & 1 & --- & --- & --- & --- & --- & 2 \\
\hline Denticulado & 1 & --- & --- & --- & --- & --- & --- & 1 \\
\hline Biface & --- & 1 & --- & --- & --- & 1 & --- & 2 \\
\hline Punta & --- & 1 & --- & --- & --- & --- & --- & 1 \\
\hline FAF & --- & --- & 1 & --- & --- & --- & --- & 1 \\
\hline TOTAL & 24 & 16 & 5 & 4 & 1 & 1 & 7 & 58 \\
\hline & & lente $\mathrm{Bla}$ & 1. Hallazgos ais & dos. Supe & elevada: & $0 \mathrm{~m}^{2}$ & & \\
\hline Tipo artefactual & & & & aterias $\mathrm{pr}$ & ticas & & & \\
\hline & & $\mathrm{Dac}$ & & & & RGFO & & \\
\hline L. externa & & - & & & & 1 & & \\
\hline L. interna & & 1 & & & & --- & & \\
\hline & Puente $\mathrm{BI}$ & o. Perfil & Superficie rel & da: $2 \mathrm{~m}^{2}$ & hos de & a sin taló & n=1) & \\
\hline Tipo artefactual & & & Materi & primas lít & & & & TOTAL \\
\hline & & acita & & cedonia & & Granit & & \\
\hline L. interna & & 4 & & 1 & & --- & & 5 \\
\hline Bola & & --- & & --- & & 1 & & 1 \\
\hline TOTAL & & 4 & & 1 & & 1 & & 6 \\
\hline & Puent & lanco 2. & rficie relevada: & $\mathrm{m}^{2}$ (dese & de talla s & talón, $n=$ & & \\
\hline Tipo artefactual & & & Materi & primas lit & & & & TOTAL \\
\hline & & $\mathrm{Dac}$ & & & $\mathrm{RG}$ & & & \\
\hline L. externa & & 5 & & & 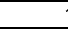 & & & 6 \\
\hline L. interna & & 4 & & & 3 & & & 46 \\
\hline Núcleo & & 5 & & & - & & & 5 \\
\hline Hoja & & 3 & & & - & & & 3 \\
\hline Cuchillo & & 1 & & & - & & & 1 \\
\hline Denticulado & & 1 & & & 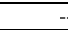 & & & 1 \\
\hline FAF & & - & & & 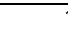 & & & 1 \\
\hline TOTAL & & 5 & & & 5 & & & 63 \\
\hline & & & e Blanco 3. Su & ficie reles & $50 \mathrm{~m}^{2}$ & & & \\
\hline Tipo artefactual & & & Materi & primas lít & & & & TOTAL \\
\hline & & & & & & & & \\
\hline Hoja & & & & & & & & 1 \\
\hline Denticulado & & & & & & & & 1 \\
\hline Raedera & & & & & & & & 2 \\
\hline TOTAL & & & & & & & & 4 \\
\hline & Puen & Blanco 4 & erficie relevada & $9 \mathrm{~m}^{2}$ (des & de talla & talón, $n=$ & & \\
\hline Tipo artefactual & & & Materi & primas lit & & & & TOTAL \\
\hline & & & & & & & & \\
\hline L. externa & & & & & & & & 4 \\
\hline L. interna & & & & & & & & 4 \\
\hline Denticulado & & & & & & & & 1 \\
\hline Cepillo & & & & & & & & 1 \\
\hline Biface & & & & & & & & 1 \\
\hline TOTAL & & & & & & & & 11 \\
\hline & Puen & Blanco 5 . & erficie relevada & $8 \mathrm{~m}^{2}$ (des & de talla & talón, $n=$ & & \\
\hline Tipo artefactual & & & Materi & primas lít & & & & TOTAL \\
\hline & & acita & & RGFO & & adera silic & cada & \\
\hline L. interna & & 3 & & 6 & & 1 & & 10 \\
\hline Núcleo & & 1 & & --- & & --- & & 1 \\
\hline Punta & & --- & & 1 & & --- & & 1 \\
\hline TOTAL & & 4 & & 7 & & 1 & & 12 \\
\hline
\end{tabular}

Tabla 1. Lugares muestreados. Tipos de artefacto por materia prima lítica. L: lasca, Indet.: indeterminada.

Table 1. Samples places. Tool types by lithic raw material. L: flake, Indet.: indeterminate.
En Puente Blanco 1 (PB1) (Tablas 1 y 2, Figura 3 A) predominan las lascas internas, entre ellas dos de arista en dacita y en RGFO y una de reducción bifacial en sílice. Dos de las lascas de calcedonia están alteradas térmicamente. En todas las rocas el $73 \%$ de los talones son no preparados, en su mayoría son lisos. Se recuperaron cuatro núcleos en dacita: son globulosos (dos tienen el $50 \%$ de corteza), tienen extracciones multidireccionales y sus ejes mayores oscilan entre 78 y $107 \mathrm{~mm}$. Hay otro sobre RGFO; que también es globuloso y con extracciones multidireccionales y cuyo eje mayor es de $35 \mathrm{~mm}$. Los artefactos formatizados $(n=10)$ corresponden tanto a filos cortos como largos, una punta de proyectil asignable al diseño Magallanes IV con un esbozo de aserrado en RGFO PKA y claras evidencias de reactivación (Figura 4 A 1), dos bifaces fracturados en su longitud en estadio 3 (Nami 1986) y un denticulado sobre lasca angular de dacita (51,5 x 35 × 9,5 mm) (Figura 4 B 1), Además, se recogió un fragmento de un artefacto (¿hebilla?) en peltre.

A partir del análisis de los restos óseos de guanaco se determinó un NISP de 71. El 63,38 \% corresponde a especímenes de ambas extremidades y un 15,59\% a articulaciones. La meteorización se estableció sobre 58 especímenes. El 81,03 \% está en el estadio 2 y el 10,34 $\%$ en estadio 1. Dos especímenes tienen hoyuelos de 

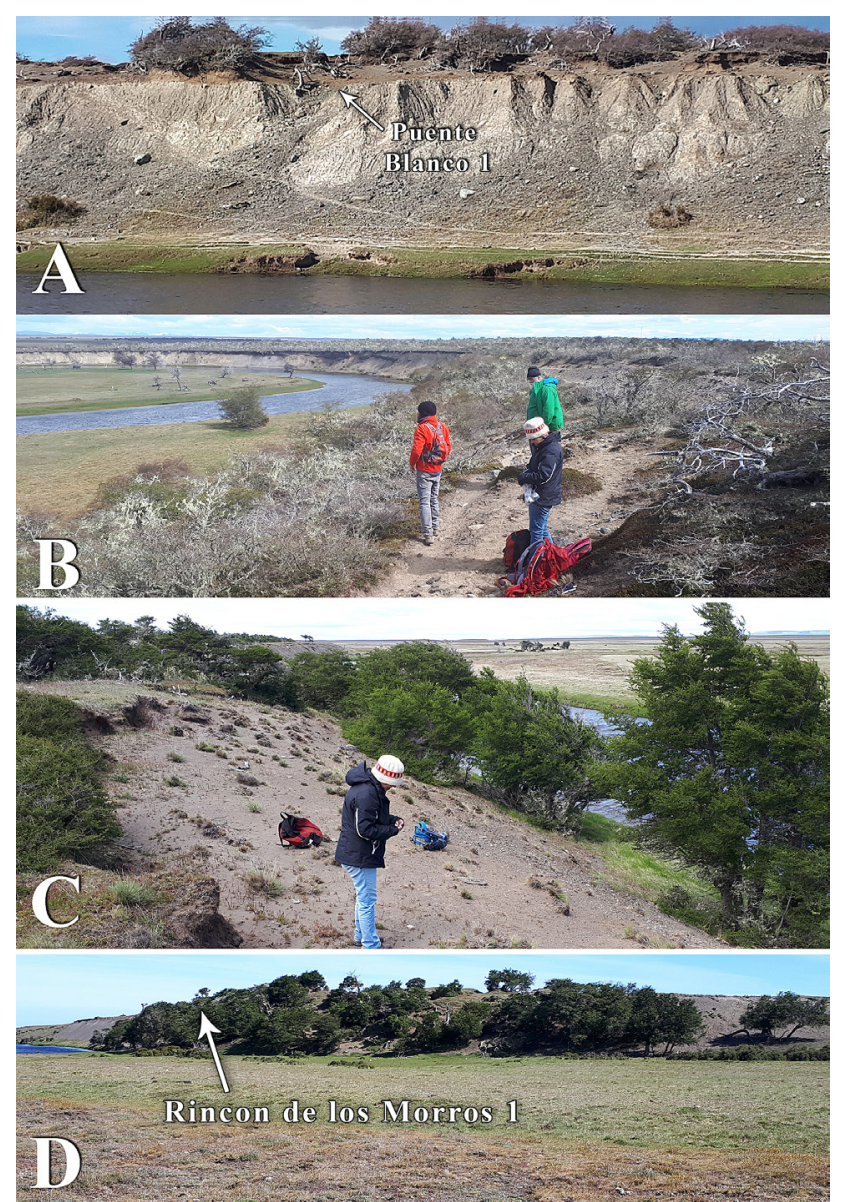

Figura 3. A. Vista de la ubicación de los sitios de Puente Blanco 1, B. Puente Blanco 5, C. Rincón de Los Morros 2 y D. Rincón de Los Morros 1.

Figure 3. A. View of sites location at Puente Blanco 1, B. Puente Blanco 5, C. Rincón de Los Morros 2 and D. Rincón de Los Morros 1.

disolución que sugieren la existencia de encharcamiento y otro, huellas de corte. Se dató una diáfisis de húmero (meteorización en estadio 2) con un negativo de impacto recuperada en superficie y asociada a los artefactos en $550 \pm 50$ años AP (LP-3288).

En Puente Blanco 2 (PB2) (Tablas 1 y 2), también son mayoría las lascas internas, entre ellas las angulares $(n=39)$. El 78\% de los talones es no preparado. Al igual que en PB1 hay cinco núcleos de dacita, globulosos y con extracciones multidireccionales. Sus ejes mayores están entre 78 y $115 \mathrm{~mm}$ Se destacan tres hojas de dacita (la única entera tiene $75 \mathrm{~mm}$ de largo) y cinco lascas de arista, cuatro de dacita y una de RGFO. Además, se registró un denticulado sobre lasca angular de dacita (70 x $44 \times 12$ $\mathrm{mm}$ ) (Figura 4 B 3).

Entre los sitios PB1 y PB2 se observó un perfil expuesto en sedimento eólico, parte se había desprendido y caído en la base del mismo. Aquí se detectó una hemibola con surco ecuatorial manufacturada en granito y desechos de talla en dacita y calcedonia. Al sitio se lo denominó Perfil caído (Tabla 1).
En Puente Blanco 3 (PB3) (Tabla 1) se recuperó una raedera doble convergente sobre hoja $(205 \times 47 \times 11 \mathrm{~mm})$ en RGFO muy alterado por pátina (Figura 4 B 6), otra hoja en dacita y un denticulado sobre una lasca primaria de dacita (77 x $45 \times 7,5 \mathrm{~mm}$ ) (Figura 4 B 4).

En Puente Blanco 4 (PB4) hay igual frecuencia de lascas internas y externas (Tablas 1 y 2). El $89 \%$ de los talones no tienen preparación. Sobresale un denticulado sobre una lasca secundaria de dacita $(74 \times 59 \times 32 \mathrm{~mm})$ (Figura 4 B 2), un cepillo de dacita (58 x $62 \times 49 \mathrm{~mm})$-conserva un $25 \%$ de corteza-, y un bifaz $(49 \times 45 \times 11,5 \mathrm{~mm})$ de RGFO en estadio 2 (Nami 1986).

Puente Blanco 5 (PB5) es el sitio que se encuentra más al este del paraje homónimo (Figura 3 B). Se encontró una punta de proyectil manufacturada sobre RGFO PKA, asignable al diseño Magallanes IV (Figura 4 A 2) y un núcleo globuloso de dacita con extracciones multidireccionales y $50 \%$ de corteza $(102 \times 67 \times 42 \mathrm{~mm})$. Todas las lascas son internas. (Tablas 1 y 2 ).

\section{Ea. Rincón de los Morros}

Estos contextos arqueológicos también se encuentran sobre el borde de la terraza en hoyadas de deflación entre manchones de ñires (Tabla 1, Figura 2, Figura 3 C y D). En el sitio Rincón de Los Morros 2 (RM2) (Tablas 1 y 2, Figura 3 C) priman las lascas internas con talones preparados y un biface fracturado en estadio 2 (Nami 1986). Se destaca que toda la muestra está conformada por RGFO, con la excepción del percutor discoidal usado en todas las caras $(96,5 \times 73 \times 55,5 \mathrm{~mm})$.

Rincón de Los Morros 1 (RM1) está conformado por concentraciones de artefactos a lo largo de unos 230 $\mathrm{m}$ (Tablas 1 y 2, Figura 3 D). Es el sitio que presenta la mayor riqueza artefactual. Molinari (2000) recuperó 14 raederas confeccionadas sobre basaltos, sílices, andesita y calcedonia -una de ellas está manufacturada sobre una hoja de basalto con filos complementarios (101 x $29 \times$ $7 \mathrm{~mm}$ ) y otra en calcedonia con corteza (55 x $30 \times 13$ $\mathrm{mm})$-, tres raspadores en sílice y calcedonia, tres puntas de proyectil triangulares con pedúnculo en basalto y un núcleo de sílice.

A continuación se describen los desechos de talla y otros artefactos de las mismas clases recuperadas por Molinari hallados en nuestros relevamientos. Priman las lascas angulares con talones sin preparar y los filos largos en RGFO y dacita. Se registraron tres puntas de proyectil de diseño Magallanes IV confeccionadas sobre RGFO -una es PKA- (Figura 4 A 3-5). Los ejemplares 3 y 4 presentan fracturas por impacto, mientras que el 5 es aserrado. Otras tres puntas son de diseño Magallanes $V$-que se destacan por sus reducidas dimensiones-, confeccionadas sobre calcedonia, RGFO PKA y obsidiana verde (Figura 4 A 6-8). Hay un biface fracturado en RGFO en estadio 3, uno en dacita $(65 \times 52,5 \times 16 \mathrm{~mm})$ en estadio 3 y otros 

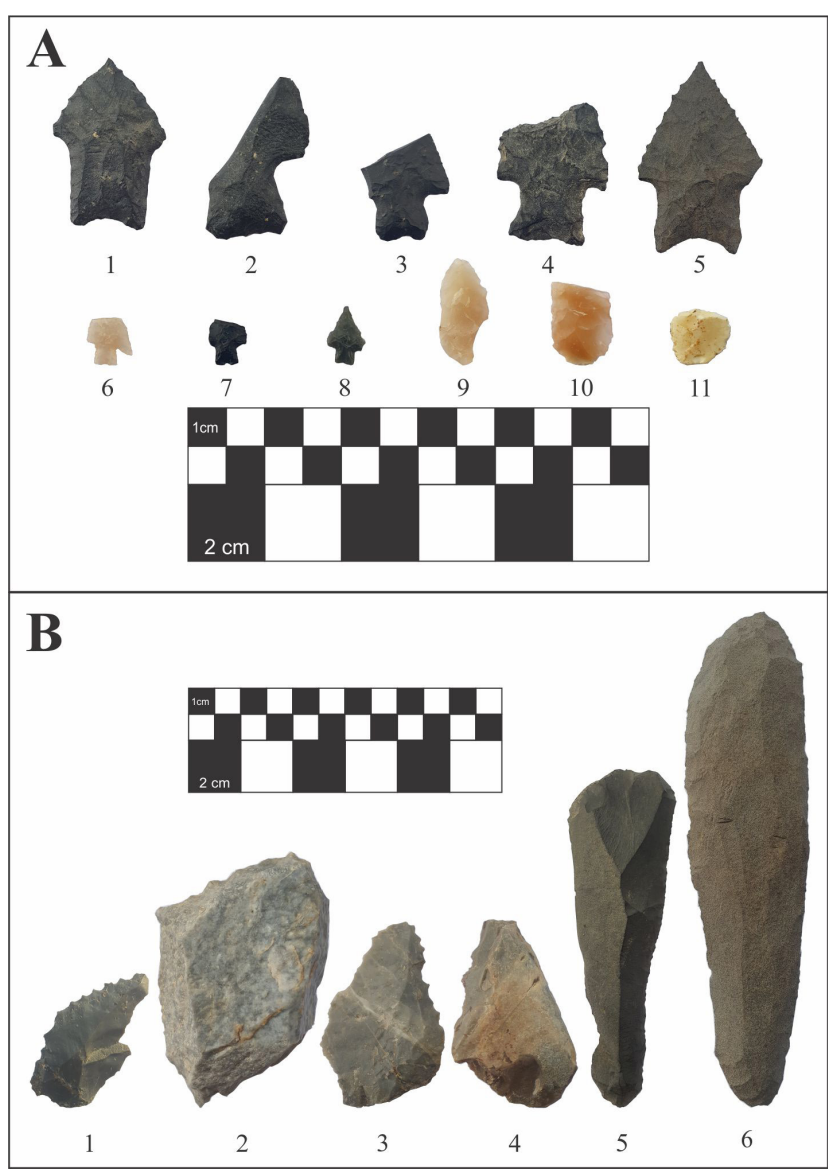

Figura 4. Muestra de artefactos. A. 1-5: Puntas de proyectil diseño Magallanes IV. Nótese que la punta 1 tiene bordes denticulados esbozados y la punta 5 tiene bordes denticulados destacados, 6-8: Puntas de proyectil diseño Magallanes $\mathrm{V}$, 9-10: Bifaces de calcedonia, 11: raspador de calcedonia. B. 1-4 artefactos denticulados, 5-6 raederas sobre hojas.

Figure 4. Sample of tools. A. 1-5: Magallanes IV projectile points design. Notice that point 1 presents incipient denticulated edges and point 5 presents prominent denticulated edges, 6-8: Magallanes V projectile points design, 9-10: Chalcedony bifaces, 11: Chalcedony end-scraper. B. 1-4 Denticulate tools, 5-6 Side-scrapers on blades.

dos en caldedonia en estadio 4 (Nami 1986) (Figura 4, A 9 y 10); el entero mide $28,5 \times 13 \times 6 \mathrm{~mm}$. Entre los raspadores se destaca uno de reducidas dimensiones (15 $\times 16,5 \times 13,5 \mathrm{~mm}$ ) confeccionado sobre una lasca angular de calcedonia alterada térmicamente (Figura 4 A 11).

Se realizó un sondeo de $0,5 \mathrm{~m}^{2}$ sobre un perfil expuesto de un médano. Los materiales se encontraron en $10 \mathrm{~cm}$ de potencia y se recuperaron 19 desechos de talla (cuatro externos de calcedonia y 15 internos -nueve de dacita, cuatro de calcedonia y uno de RGFO), un raspador de calcedonia y una raedera en RGFO (ambos fracturados). Se identificaron 12 especímenes de guanaco con predominio de articulaciones y astillas de huesos largos. El 83,33 $\%$ se encuentra en el estadio 1 de meteorización y hay un fragmento de metacarpo en estadio 3. Además, se regularizó un perfil expuesto sobre otro médano ubicado al oeste del sitio, hacia el final de la distribución de materiales. Se recuperaron articulados un metatarso proximal con huellas de corte, el calcáneo proximal, el astrágalo y cuatro articulaciones de guanaco (todos en estadio de meteorización 1). Se dató el $4^{\circ}$ tarsiano en $403 \pm 36$ años AP (AA113564).

\section{Ea. Rincón de Los Morros-Lagunas}

Son siete lagunas de régimen temporal que exponen rodados de variada litología y tamaños (Tabla 1, Figura 2). Se recorrieron sus perímetros, único espacio sin cubierta vegetal. Sólo se observaron dos artefactos líticos en las lagunas 5 y 7 .

\section{Bosque de las Bandurrias}

El relevamiento sumario dio como resultado muy baja frecuencia de artefactos (Tabla 1, Figura 2). Es de destacar un artefacto compuesto manufacturado en calcedonia sobre una lasca de arista de módulo laminar -tiene un filo largo de cuchillo y otro frontal corto de raspador $(53,5 \times 22 \times 6 \mathrm{~mm})$.

\section{Discusión y conclusiones}

Las dataciones radiocarbónicas obtenidas en los sitios PB 1 (550 \pm 50 años AP) y RM1 (403 \pm 36 años AP) se alinean con la obtenida en Morro Chico (480 \pm 30 -Alfonso-Durruty et al. 2015) y profundizan la cronología obtenida en laguna Cóndor, en torno a los 200 años AP (L'Heureux y Borrero 2016). Más allá de esta diferencia se refuerza la ocupación eminentemente tardía de la zona de los morros (Borrero 2015; Borrero et al. 2011; Charlin et al. 2011), coincidente con la PEH, lo que como mínimo debió afectar los patrones de circulación microrregionales debido a la mayor estacionalidad y cobertura arbórea. Estos cambios pudieron limitar la accesibilidad y utilización del río Penitente durante períodos más largos dentro del ciclo anual.

El registro arqueológico del curso inferior del río Penitente se concentra sobre el bosque de la terraza de la margen este de los sectores de Puente Blanco y Rincón de los Morros y tiene una distribución relativamente continua; el análisis de la Tabla 2 permite comparar los contextos más importantes. Ni las lagunas de Rincón de Los Morros ni el Bosque de Las Bandurrias presentan frecuencias y riquezas altas de artefactos, por lo que son interpretados como espacios donde primó la circulación por sobre la instalación.

El sitio PB6 es el de mayor densidad artefactual, seguido por PB2 (Tabla 1). Según la riqueza, los sitios se agrupan en tres conjuntos: PB6 (una sola clase -lascas), PB5, PB4 y RM 2 (tres y cuatro clases) y PB2 y PB1 y RM1 (con seis y ocho clases, respectivamente). Las diferencias son entendidas como el reflejo de ocupaciones de distinta intensidad y funcionalidad. Las tareas de talla (que 


\begin{tabular}{|c|c|c|c|c|c|c|c|c|}
\hline & \multirow{2}{*}{\multicolumn{7}{|c|}{ Sitios }} \\
\hline & & & & & & & & \\
\hline & & \multicolumn{5}{|c|}{ Puente Blanco } & \multicolumn{2}{|c|}{$\begin{array}{l}\text { Rincón de Los } \\
\text { Morros }\end{array}$} \\
\hline & & PB6 & PB1 & PB2 & PB4 & PB5 & RM2 & RM1 \\
\hline \multicolumn{2}{|c|}{ Densidad $\mathrm{m}^{2}\left(10^{-1}\right)$} & 5 & 0,27 & 0,81 & 0,15 & 0,20 & 0,29 & $0,12^{*}$ \\
\hline \multicolumn{2}{|c|}{ Riqueza } & 1 & 8 & 6 & 4 & 3 & 4 & 8 \\
\hline \multicolumn{2}{|c|}{$\begin{array}{l}\text { Relación núcleos / desechos de } \\
\text { talla }\end{array}$} & --- & 0,11 & 0,09 & --- & 0,1 & --- & 0,02 \\
\hline \multicolumn{2}{|c|}{$\begin{array}{l}\text { Relación desechos de talla } \\
\text { externos / internos }\end{array}$} & 0,25 & 0,65 & 0,12 & 1 & --- & --- & 0,21 \\
\hline \multicolumn{2}{|c|}{$\begin{array}{l}\text { Relación artefactos } \\
\text { formatizados / desechos de talla }\end{array}$} & --- & 0,23 & 0,05 & 0,37 & 0,1 & 0,22 & 0,22 \\
\hline \multirow{3}{*}{$\begin{array}{l}\text { \% de materias } \\
\text { Primas }\end{array}$} & Dacita & --- & 41,37 & 92,06 & 63,63 & 33,33 & --- & 31,25 \\
\hline & RGFO & 100 & 27,58 & 7,93 & 36,36 & 58,33 & 90,90 & 52,67 \\
\hline & Calcedonia & --- & 8,62 & --- & --- & --- & --- & 13,39 \\
\hline
\end{tabular}

Tabla 2. Variables de análisis por sitio. * Se suman los 21 artefactos formatizados relevados por Molinari (2000).

Table 2. Analytical variables per site. * 21 formatted tools recorded by Molinari (2000) are added.

incluyen desbaste inicial) son más importantes en PB1 y PB2, lo que se refleja en la mayor proporción de núcleos y desechos de talla externos. El primero también tiene lascas relacionadas con la manufactura de bolas. Los demás también presentan indicadores de tareas de talla pero no focalizadas sobre las etapas iniciales de desbaste, lo que se podría relacionar con el desarrollo de etapas intermedias de talla y la entrada a los sitos de artefactos manufacturados. El sitio PB4 posee la proporción más alta de artefactos formatizados y desechos de talla, aunque puede deberse a un problema del tamaño de la muestra. Tres sitios, PB1, RM2 y RM1, tienen valores similares, no obstante estos dos últimos difieren entre sí en las frecuencias y tipos de artefactos formatizados. Sobre un espectro de espacios donde se habrían desarrollado tareas limitadas, se destacan PB1 y PB2 por la riqueza artefactual, que sugiere el desarrollo de un abanico más amplio de actividades. Además, PB1 presenta arqueofauna de guanaco. Es llamativa en términos regionales la alta frecuencia de bifaces $(\mathrm{N}=8)$. Todo lo señalado es aún más notorio en RM1. Las concentraciones de menos de 10 artefactos (Tabla 1), con un fragmento de bola, uno de los denticulados (Figura 4 B 4) y una de las raederas sobre hoja (Figura 4 B 6), terminan de configurar un marco de mayor diversidad.

Hay otro aspecto que realza a los conjuntos de Puente Blanco y al sitio RM1: su ubicación en el paisaje del sur de los morros. Los sitos PB1 y PB2 (PB3, PB4 y PB6 acompañan por su cercanía) se encuentran frente a la confluencia de los ríos Penitente y Rubens, este último proviene del sudoeste y puede ser también una ruta de circulación hacia y desde la zona; por su parte, RM1 se ubica justo en el sector de terraza donde el río Penitente cambia su curso hacia el oeste y donde el bosque tiene su distribución más oriental. Esto hace que el sector de terraza donde se encuentra el sitio sea claramente identificable si se está circulando desde el sur hacia el río Gallegos (Figuras 1 y 2).

Con respecto a las materias primas líticas utilizadas, en los sitios ubicados más hacia el este (RM2 y RM1), priman las RGFO, mientras que, en los localizados hacia el oeste -sitios PB1 a 6- dominan las dacitas (Tablas 1 y 2). Esto se ajusta a lo observado en el CVPA, donde si bien las dacitas son las rocas más abundantes y de amplia distribución espacial, ocupan el segundo lugar en la elección de materias primas luego de las RGFO, frecuentes en el interfluvio Gallegos-Chico (Charlin 2009, Charlin et al. 2011, entre otros). La calcedonia ocupa el tercer lugar en la jerarquía de uso, al igual que en el CVPA (Charlin 2009). Además, sólo tiene una representación destacada en PB1 y RM1; en los demás registros lo hace en muy baja frecuencia (Tabla 1). Los materiales recuperados en el sondeo de RM1 mantienen estas tendencias. Se destaca el instrumento unifacial de filos compuestos del BB ya que son característicos del CVPA (Charlin 2009). También se registraron una punta de proyectil, dos bifaces y un raspador (Figura 4 A 6, 9,10 y 11, respectivamente). En la cueva La Carlota y en el alero Barría (margen sur del curso medio del río Gallegos) se han observado artefactos de reducidas dimensiones que se habrían confeccionado a partir de tabletas inmediatamente disponibles (Campan et al. 2007). El predominio de núcleos bipolares en el sector norte del CVPA es coincidente tanto con dicha disponibilidad como con los tamaños en que se presenta (Charlin 2009). Si bien en muestreos de rocas llevados a cabo en el interior del CVPA sólo se registró un nódulo de calcedonia (19 mm en su eje mayor), la mayor frecuencia de artefactos sugiere que esta materia prima también se encontraría disponible en el sector central (Eas. Don Bosco y Cóndor) (Charlin 2009). Recientemente, en la margen norte de la cuenca media del río Gallegos se identificó una alta frecuencia de desechos de talla de calcedonia traslúcida en conjuntos recuperados en Campo Las Piedras (Ea. Bella Vista) y en la margen oeste de la laguna Alquinta, lindante con el cañadón Mack Aike o Chorrillo Grande (Ea. Alquinta), 60-70 km al noreste de Puente Blanco, respectivamente. Los ejes mayores de los núcleos oscilan entre los 30 y los $70 \mathrm{~mm}$ y tienden a ser de forma esferoidal (Carballo Marina y Belardi 2018), lo que se relacionaría con la formación de la calcedonia en vesículas volcánicas (Charlin 2009). Estas observaciones refuerzan los planteos previos acerca de la disponibilidad de calcedonia en el sector norte del CVPA (Campan et al. 2007; Charlin 2009). Entonces, si bien se desconoce el lugar preciso de proveniencia de esta roca, todo sugiere su ingreso a los morros desde el este $y$, dadas las dimensiones 
de los artefactos de filo largo de RM1 y BB, no provendría exclusivamente de tabletas.

En el curso inferior del río Penitente hay raederas manufacturadas sobre hojas de lutita que muestran similitud morfológica con aquellas asignadas al núcleo poblacional del noroeste (Pallo et al. 2020). Si bien en baja frecuencia $(\mathrm{N}=2)$ estos artefactos se registraron en los contextos de Puente Blanco. La procedente de PB6 (103 x $41 \times 10 \mathrm{~mm}$ ) (Figura 4 B 5) se ajusta a la media de este tipo de artefactos, mientras que la de PB3 (205 x $47 \times 11 \mathrm{~mm})$ (Figura 4 B 6) posee una longitud mayor a las conocidas tanto al oeste como al este del río Penitente (Pallo et al. 2020, Figura 2). Hay otra raedera, manufacturada en basalto, que fue informada por Molinari (2000) en el sitio RM1 que se ajustaría a la descripción de este tipo de artefactos, mide $101 \times 2 \times 7$ mm. Las raederas confeccionadas sobre grandes formas base laminares han alcanzado también la cuenca media del río Coyle, a unos $100 \mathrm{~km}$ hacia el noreste del río Penitente, ya que se registró un ejemplar sobre RGFO (137 x $40 \times 12 \mathrm{~mm})$ en el sitio Rubén Aike (Carballo Marina et al. 2000-2002). Se debe destacar que el Brazo Norte del Coyle es la ruta natural de circulación por la que se accede desde el este a los sectores con materias primas de Ea. La Verdadera Argentina.

Los conjuntos del río Penitente poseen puntas de proyectil asignables a los diseños Magallanes IV y V , de circulación usual en el sur de Patagonia continental durante el Holoceno tardío (Bird 1993; Cirigliano 2011; Gómez Otero 1986-1987; Franco et al. 2005). Las puntas han sido manufacturadas sobre RGFO (concordante con la mayor frecuencia de bifaces $-n=5$ ), RGFO PKA, dacita, calcedonia y obsidiana verde. Sobre la base del ancho de los pedúnculos, las puntas de diseño Magallanes IV (Figura 3 A 1-5) corresponderían a sistemas de armas arrojadizas mientras que las Magallanes $\mathrm{V}$ a arco y flecha (Figura $4 \mathrm{~A}$ 6-8) (Ratto 1991). Entre las puntas de proyectil Magallanes IV sobresalen dos con bordes dentados/aserrados (un ejemplar con esbozos -Figura 4 A 1-y en otro claramente definido -Figura 4 A 5-, característica también observada en una punta Magallanes IV manufacturada en RGFO registrada en la laguna Little Hill (observaciones recientes). Dado que el aserrado no afectaría de manera significativa ni el rendimiento ni el funcionamiento de la punta en la perforación de distintas sustancias, se lo ha vinculado con connotaciones identitarias o simbólicas (Loendorf et al. 2015). No obstante, se ha destacado que el borde aserrado serviría para desgarrar heridas (Smallwood et al. 2018). La baja frecuencia en que se presenta el aserrado en puntas de proyectil de diseño triangular del sur de Patagonia (por ejemplo, Bird 1993, Figuras 14 y 15 -en puntas diseño Magallanes III; Espinosa et al. 2020, Figura 2 b -punta diseño Magallanes IV) sugiere que, si bien el aserrado puede tener un valor funcional, no es determinante para el desempeño de las puntas. Dado que la regularización del filo aserrado parece requerir de un trabajo de precisión realizado hacia la finalización de los bordes, el aserrado podría acarrear un componente identitario individual.

También se han registrado artefactos unifaciales con filo aserrado/dentado ( $\mathrm{N}=4$ ) (Figura 4 B 1-4) en Puente Blanco -donde los dientes están más separados y donde se infiere una menor necesidad de precisión en la talla respecto de las puntas de proyectil. El contexto más cercano al río Penitente con presencia de denticulados $(n=1)$ es el sitio Cerro León 3, en el sudeste del cordón Baguales (Borrazzo 2006). La mayor frecuencia se ha registrado en la costa norte de Santa Cruz; estudios experimentales indicaron que serían adecuados para trabajar el hueso en estado fresco y seco, sin buen desempeño sobre madera (Ambrústolo et al. 2010). Esto es relevante dado que los artefactos denticulados unifaciales son el único componente artefactual del río Penitente que difiere de los reconocidos en la estepa en general. Queda abierta la discusión de su funcionalidad. Las localizaciones de estos hallazgos, dentro de un corredor de comunicación con el sur, hacen atractiva su consideración conjunta con varios otros marcadores de interacción con las zonas costeras (Borrero y Barberena 2006).

Las relaciones con el sur, específicamente con el mar de Otway, se manifiestan en la circulación de obsidiana verde (Morello et al. 2015). Esta roca se empleó para la manufactura de una punta de proyectil diseño Magallanes $\mathrm{V}$ en RM1 (Figura 4 A 8). A ella se suman las lascas que acompañan el enterratorio de Morro Chico (Prieto 1984) y la recuperada en la laguna Cóndor (Charlin 2012). Además, se identificaron tres lascas en la laguna Little Hill, la más grande de ellas de 41 x 33,5 x 11,5 $\mathrm{mm}$ (observaciones personales). La vinculación con la costa también queda en evidencia por la dieta mixta del individuo del enterratorio de Morro Chico (AlfonsoDurruty et al. 2015) y la presencia del molusco en morro Philippi (Ortiz Troncoso 1973). La obsidiana verde habría circulado por la estepa en dirección norte, por donde se llega sin dificultad al valle del río Penitente e ingresaría a la zona de los morros. Lo señalado refuerza el papel del río Penitente como vía de circulación. La obtención de esta roca, presente en baja frecuencia, no es necesariamente resultado de rangos de acción muy amplios, sino más bien de redes de circulación de individuos portadores de esos materiales (Borrero et al. 2011).

El paisaje arqueológico del curso inferior del río Penitente amplía la información de la zona de los morros al sumar variabilidad a la discusión acerca del uso humano de este espacio. Presenta una relativa representación espacial y diversidad de contextos de ocupación que es mayor al esperado bajo un uso estrictamente mortuorio y/o logístico. Las ocupaciones muestran componentes residenciales, redundancia genérica en el uso de la terraza de la margen este del río, y posiblemente específica en el caso de los sitios de Puente Blanco (PB 1 y PB2), donde 
se produce la confluencia con el río Rubens. También en el sitio RM1, donde el río cambia de dirección hacia el oeste. En estos puntos las densidades artefactuales son más altas, en un orden de magnitud de $10^{-1}$ (Tabla 2). Esto concuerda con la idea de ver al río como un corredor convergente con el curso principal del río Gallegos y vía de acceso al interior y son señales que impiden considerar a la cuenca como un mero atractor de ocupaciones circunstanciales, o ruta exclusivamente de paso. El paisaje arqueológico sugiere, en cambio, un espacio de uso regular, que seguramente implica grados de planificación que avalan la sistematicidad en el uso de la cuenca. En una escala espacial mayor, el registro artefactual del curso inferior del río Penitente da cuenta de la interacción con los núcleos poblacionales del noroeste y del este (CVPA), el solapamiento de sus respectivos rangos de acción, y muestra la conexión de los espacios del sur (mar de Otway) con el sector de los morros durante el Holoceno tardío. Esto no hace más que resaltar los planteos sobre el papel central de los morros tanto en términos ideacionales, relacionados con los contextos mortuorios, como así también con su relevancia para los cazadores al ser hitos en el paisaje (Borrero et al. 2011). En este marco de interacciones, el uso específico del bosque estuvo relacionado con el reparo que brinda, ya sea tanto bajo circunstancias de circulación (Bosque de Las Bandurrias) como de mayor permanencia (terraza de Puente Blanco y Rincón de Los Morros).

Río Gallegos, 30 de junio de 2020

\section{Agradecimientos}

Al Sr. Diego Bernad (Ea. Rincón de los Morros) por su gentileza, hospitalidad e inestimable colaboración durante los trabajos de campo.

A los dueños de la estancia Glen Cross por permitirnos trabajar en su propiedad.

Las Dras. Karen Borrazzo y Silvana Espinosa, el Lic. Pedro Tiberi y el Ing. Lucas Monelos siempre estuvieron dispuestos a responder nuestras consultas. A los dos evaluadores anónimos por sus útiles comentarios y sugerencias. Al Sr. Pablo Binaghi por su colaboración en las tareas de campo.

A la Universidad Nacional de la Patagonia Austral (PIP 29A/405).

\section{Bibliografía}

Alfonso-Durruty, M. P., Giles, B. T., Misarti, N., San Román, M. y Morello, F. (2015). Antiquity and Geographic Distribution of Cranial Modification among the Prehistoric Groups of Fuego-Patagonia, Chile. American Journal of Physical Anthropology 158 (4), 607-623. doi: 10.1002/ ajpa.22832.
Ambrústolo, P., Castro, A., Zubimendi, M. A. y Mazzitelli, L. (2010). Instrumentos líticos con filos denticulados en la costa norte de Santa Cruz. Un análisis tecno-funcional. Cazadores recolectores del Cono Sur 4, 79-94.

Aragón, E. y Franco, N. V. (1997). Características de rocas para la talla por percusión y propiedades petrográficas. Anales del Instituto de la Patagonia (Serie Ciencias Humanas) 25, 187-200.

Aschero, C. (1975). Ensayo para una clasificación morfológica de los instrumentos líticos aplicada a estudios tipológicos comparativos. Informe al CONICET.

Aschero, C. (1983) Ensayo para una clasificación morfológica de los instrumentos líticos aplicada a estudios tipológicos comparativos. Apéndices A-C revisión. Cátedra de Ergología y Tecnología, Facultad de Filosofía y Letras, Universidad de Buenos Aires, Buenos Aires.

Bird, J. B. (1993). Viajes y Arqueología en Chile Austral. En J. Hyslop (Ed.). Punta Arenas, Chile: Ediciones de la Universidad de Magallanes.

Borrazzo, K. B. (2006). Tecnología lítica del alero Cerro León 3 (Santa Cruz, Argentina). Magallania 34 (2), 63-74.

Borrero, L. A. (1994-1994). Arqueología de la Patagonia. Palimpsesto. Revista de Arqueología 5, 9-69.

Borrero, L. A. (2015). Spatial Demarcation of Archaeological Population Cores in Southern Patagonia. En World Heritage Papers 42. Heads 5. Human Origin Sites and the World Heritage Convention in the Americas, Volume 1 (pp. 211-218). Ciudad de México: UNESCO y World Heritage Convention.

Borrero, L. A. y Barberena, R. (2006). Hunter-gatherer home ranges and marine resources. Current Anthropology 47(5), 855-67.

Borrero, L. A. y Borrazzo, K. (2011). La geografía cultural del sudoeste de Patagonia continental. En L. A. Borrero y K. Borrazzo (Comps.), Bosques, Montañas y Cazadores. Investigaciones Arqueológicas en Patagonia Meridional, (pp. 7-36). Buenos Aires: CONICET-IMHICIHU.

Borrero, L., Martin, A. F. y Barberena, R. (2011). Visits, "Fuegians" and Information Networks. En R. Whallon, W. Lovis y R. Hitchcock (Eds.), Information and Its Role in Hunter-Gatherer Bands. Ideas, Debates, and Perspectives 5, (pp: 249-265). California: Cotsen Institute of Archaeology Press.

Campan, P., Carballo Marina, F. y Manzi, L. (2007). Arqueología de Estancia La Carlota (Campo volcánico Pali Aike, Argentina). En F. Morello, M. Martinic, A. Prieto y G. 
Bahamonde (Eds.), Levantando piedras, desenterrando huesos... y develando arcanos, (pp. 687-699). Punta Arenas: Ediciones CEQUA.

Carballo Marina, F., Belardi, J. B., Espinosa, S. y Ercolano, B. (2000-2002). Tecnología y movilidad en el río Coyle, Santa Cruz. Cuadernos del Instituto Nacional de Antropología y Pensamiento Latinoamericano 19, 89-107.

Carballo Marina, F. y Belardi, J. B. (2018). Distribución espacial del registro arqueológico localizado en el polígono de registración sísmica 3D "El Turbio Este", departamento Güer Aike, provincia de Santa Cruz. Informe entregado a la Empresa ENAP-SIPETROL, Argentina. Ms

Carballo Marina, F., Belardi, J. B. y Borrero, L. A. (2016). Nuevos datos para la discusión arqueológica de corredores en el bosque del suroeste de la provincia de Santa Cruz, Argentina: el caso del arroyo Los Loros. Magallania, 44 (2), 209-217.

Carballo Marina, F., Manzi, L., Campan, P., Belardi, J. B., Tiberi, P., Manero, A. y Sáenz, J. L. (2008). Distribución del registro arqueológico en la cuenca del río Gallegos (Santa Cruz): línea de base y aporte a la preservación del patrimonio. En L. A. Borrero y N. V. Franco (Eds), Arqueología del Extremo sur del Continente Americano, (pp.175-225). Buenos Aires: CONICET-IMHICIHU.

Charlin, J. (2005). Aprovisionamiento de materias primas líticas en el campo volcánico de Pali Aike (Santa Cruz): una primera aproximación a partir del análisis de los núcleos. Werken 7 (2), 39-55.

Charlin, J. (2009). Estrategias de Aprovisionamiento y Utilización de las Materias Primas Líticas en el Campo Volcánico Pali Aike (Provincia de Santa Cruz, Argentina). British Archaeological Reports. Oxford, Inglaterra: Archaeopress.

Charlin, J., Borrero, L. A. y Pallo, C. (2011). Ocupaciones humanas en el área noroccidental del río Gallegos (Prov. Santa Cruz, Argentina). En L. Borrero y K. Borrazzo (Eds.), Bosques, montañas y cazadores. Investigaciones arqueológicas en Patagonia meridional, (pp. 179-210). Buenos Aires: CONICET-IMHICIHU.

Charlin, J. (2012). Materias primas líticas y uso del espacio en las nacientes del río Gallegos: el caso de laguna Cóndor (Estancia Glencross, Santa Cruz, Argentina). Magallania 40, 163-184. doi: 10.4067/ S0718-22442012000100010

Childs, H. (1936). El Jimmy, A Patagonian Outlaw. Philadelphia-London: J. B. Lippincott Company.

Cirigliano, N. A. (2011). Materias primas líticas y cronologías de puntas pedunculadas tipo Fell V entre las cuencas de los ríos Chico -curso inferior y medio- y Santa Cruz (provincia de Santa Cruz). La Zaranda de Ideas. Revista de Jóvenes Investigadores en Arqueología 7, 9-22.

Cirigliano, N. A, Pallo, M. C. y Charlin, J. E. (2020). Explotación y circulación de rocas de grano fino oscuras tipo Potrok Aike: el análisis de dos conjuntos líticos de Patagonia extremo sur continental (Argentina). Revista del Museo de Antropología 13 (1), 197-202.

Díaz, B., Monserrat, M. C., Tiberi, P., Mardewald, G., Hofmann, Ch., Caparrós, Mattenet F., Zerpa, D., Billoni, S. L. y Martínez, L. (2015). Hidrología superficial de la Región Hidrográfica del río Gallegos (Sur de la provincia de Santa Cruz, Argentina). https:// publicaciones.unpa.edu.ar/index.php/ICTUNPA/article/ view/697/365

Dixie, F. (1996). A través de la Patagonia. Punta Arenas, Chile: Ediciones de la Universidad de Magallanes.

Dunnell, R. C. y Dancey, W. (1983). The siteless survey: A regional scale data collection strategy. En M. Schiffer (Ed.), Advances in Archaeological Method and Theory 6: 267-287. New York: Academic Press.

Espinosa, S. L, Belardi, J. B. y Carballo Marina, F. (2020). Puntas de proyectil líticas de la cuenca media e inferior del río Coyle (Santa Cruz): aportes al estudio del uso del espacio regional y a la distribución espacial del diseño Magallanes IV. En prensa.

Foley, R. (1981). Off-Site Archaeology and Human Adaptation in Eastern Africa. An Analysis of Regional Artefact Density in the Amboseli, Southern Kenia. Cambridge Monographs in African Archaeology 3. Oxford: BAR International Series 97.

Franco, N. V., Cardillo, M. y Borrero, L. A. (2005). Una primera aproximación a la variabilidad presente en las puntas denominadas"Bird IV". Werken 6 (1), 81-95.

Garibotti, I. A. y Villalba, R. (2009). Lichenometric dating using Rhizocarpon subgenus Rhizocarpon in the Patagonian Andes, Argentina. Quaternary Research 71, 271-283.

Glasser, N.F., Harrison, S., Winchester, V., Aniya, M. (2004). Late Pleistocene and Holocene palaeoclimate and glacier fluctuations in Patagonia. Global and Planetary Changes 43, 79-101.

Golledge, R. (2003). Human Wayfinding and Cognitive Maps. En M. Rockman y J. Steele (Eds.), Colonization of Unfamiliar Landscapes. The Archaeology of Adaptation, (pp. 25-43). Londres: Routledge. 
Gómez Otero, J. (1986-1987). Investigaciones arqueológicas en el alero Potrok Aike (Prov. de Santa Cruz). Una revisión de los períodos IV y $\mathrm{V}$ de Bird. Relaciones de la Sociedad Argentina de Antropología XVII (1): 173-200.

Gómez Otero, J. (1991). Discusión sobre el límite occidental del territorio de los Proto-Tehuelches y Tehuelches meridionales en el extremo Sud de Patagonia (cuenca del río Gallegos). Waxen 3, 5-21.

Greenwood, W. H. (2015). Patagonia Bravía. Naturaleza, vidas y aventuras. Memorias originales del baqueano William H. Greenwood. En G. Grace Paz y D. Campbell (Eds.) Santiago de Chile: Salesianos Impresores S. A. Huber, U. M. y Markgraf, V. (2003). European impact on fire regimes and vegetation dynamics at the steppe-forest ecotone of southern Patagonia. The Holocene 13, 567-579.

Huber, U. M., Markgraf, V. y Schäbitz, F. (2004). Geographical and temporal trends in Late Quaternary fire histories of Fuego-Patagonia, South America. Quaternary Science Reviews 23, 1079-1097.

L'Heureux, G. L. y Borrero L. A. (2016). El uso de la fauna en laguna Cóndor, provincia de Santa Cruz, Argentina. Magallania 44 (1), 249-257.

Loendorf, Ch., Oliver, T., Tiedens, S., Plumlee, R., Woodson, M. y Simon, L. (2015). Flaked-stone projectile point serration: A controlled experimental study of blade margin design. Journal of Archaeological Science: Reports 3, 437-443.

Mancini, M. V. (2002). Vegetation and climate during the Holocene in Southwest Patagonia, Argentina. Review of Paleobotany \& Palynology 122, 101-115.

Martinic, M., Prieto, A. y Cárdenas, P. (1995). Hallazgo del asentamiento del jefe Aonikenk Mulato en el Valle del Zurdo. Una prueba de la sedentarización indígena en el período histórico final. Anales del Instituto de Patagonia 23, 87-94.

Mazzoni, E. y Vázquez, M. (2001). Evaluación de pastizales húmedos para un aprovechamiento sustentable en la cuenca del río Gallegos (Provincia de Santa Cruz, Argentina). VIII Encuentro Latinoamericano de Geógrafos, (pp: 175-182). Santiago de Chile.

Mazzoni, E. (2017). Landscapes of Southern Patagonia, Argentina, Their Scenic Characteristics and Their Importance for the Geotourism. En J. Rabassa (Ed.), Advances in Geomorphology and Quaternary Studies in Argentina. Proceedings of the Sixth Argentine Geomorphology and Quaternary Studies Congress, (pp. 141-179). Suiza: Springer Earth System Sciences.
Mengoni Goñalons, G. L. (1999). Cazadores de guanaco de la Estepa Patagónica. Buenos Aires: Sociedad Argentina de Antropología.

Molinari, R. 2000. Relevamiento arqueológico en la estancia Rincón de los Morros (Santa Cruz). MS.

Morello, F., Stern, Ch. y San Román, M. (2015). Obsidiana verde en Tierra del Fuego y Patagonia: caracterización, distribución y problemáticas culturales a lo largo del Holoceno. Intersecciones en Antropología. Volumen Especial 2, 139-153.

Moreno, P. I., Francois, J. P. Villa-Martinez, R. P. y Moy, C. M. (2009). Millennial-scale variability in Southern Hemisphere westerly wind activity over the last 5000 years in SW Patagonia. Quaternary Science Reviews 28, 25-38.

Nami, H. (1986). Experimentos para el estudio de la tecnología bifacial de las ocupaciones tardías en el extremo sur de la Patagonia continental. Informes de Investigación PREP 5. Programa de Estudios Prehistóricos, Buenos Aires.

Ortiz Troncoso, O. (1973). Artefactos de silex de una tumba de morro Philippi, valle medio del río Gallegos (Prov. de Santa Cruz, Rep. Argentina). Anales del Instituto de la Patagonia IV (1-3), 131-139.

Pallo, C. y Borrero, L. A. (2015). Arqueología de corredores boscosos en Patagonia Meridional: el caso del río Guillermo (SO de la provincia de Santa Cruz, Argentina). Intersecciones en Antropología 16: 313-326.

Pallo, C., Cirigliano, N. A., Charlin, J. y Borrazzo, K. 2020. Una aproximación a la distribución de la producción laminar en Patagonia Meridional. Revista del Museo de Antropología 13 (1), 217-222.

Peri, P. y Ormaechea, S. (2013). Relevamiento de los bosques nativos de ñire (Nothofagusantarctica) en Santa Cruz: base para su conservación y manejo. Ministerio de Agricultura, Ganadería y Pesca. Presidencia de la Nación. INTA. Buenos Aires: Ediciones Instituto Nacional de Tecnología Agropecuaria.

Prieto, A. (1984). Hallazgo de un colgante decorado en Morro Chico (Magallanes). Anales del Instituto de la Patagonia 14, 59-61.

Ratto, N. (1991). Análisis funcional de las puntas de proyectil líticas de sitios del sudeste de la Isla Grande de Tierra del Fuego. Arqueología 1, 151-178.

Rogers, J. T. (2002). Expedición a la parte austral de la Patagonia por teniente $2^{\circ}$ de la Armada de Chile señor Juan Tomás Rogers. En M, Martinic (Ed.), Marinos de a caballo. Exploraciones terrestres de la Armada de Chile 
en la Patagonia Austral y la Tierra del Fuego 1877-1897, (pp: 19-60). Punta Arenas: Universidad de Magallanes y Universidad de Playa Ancha.

Schäbitz, F. (1991). Holocene vegetation and climate in Southern Santa Cruz, Argentina. Bamberger Geographische Schriften Bd. 11, 235-244.
Smallwood, A. M., Smith, H., Pevny, Ch. y Jennings, T. (2018). The Convergent Evolution of Serrated Points on the Southern Plains-Woodland Border of Central North America. En M. J. O`Brien, B. Buchanan y M. I. Eren (Eds.), Convergent Evolution in Stone-Tool Technology, (pp. 203-227). Vienna Series in Theoretical Biology. Cambridge: The MIT Press. 